\title{
Capillary electrophoresis for the determination of new markers of natural latex quality
}

\author{
V. Galli, N. Olmo, C. Barbas* \\ Facultad de CC Experimentales y Técnicas, Universidad San Pablo-CEU, Urbanización Montepríncipe, Ctra. Boadilla del Monte, \\ km 5.3, 28668 Madrid, Spain
}

\begin{abstract}
Nowadays, increasing use of latex products in the healthcare area has also led to an increase in new scientific controls of raw material and manufactured products in order to maintain higher standards in quality control. Since field latex is a rich environment for microbial growth, in the absence of adequate preservation, the carbohydrates that it contains become microbiologically oxidised to the so-called volatile fatty acids (VFAs). Samples of natural rubber latex coming from different countries and processed in different ways have been tested by applying a capillary electrophoresis method for the measurement of the short-chain organic acids present in sera. The separation was performed with phosphate buffer at $\mathrm{pH}$ 6.25 and cetyltrimethylammonium bromide (CTAB) as background electrolyte in an uncoated fused-silica capillary with $-10 \mathrm{kV}$ of applied potential. The sample needs no other pre-treatment more than coagulation to obtain the serum. In the assayed samples, it has been shown that poorly-preserved latex presented higher amounts of succinic acid and lower amounts of malic acid. The succinic to malic acid ratio may be an important parameter because it cannot be altered by dilution or similar processes such as the traditional VFA index, used to determine the quality of latex. Ratios for succinic to malic acid $<0.6$ have been found for well-preserved latex and $>0.6$ for poorly-preserved latex. C) 2002 Elsevier Science B.V. All rights reserved.
\end{abstract}

Keywords: Latex; Organic acids

\section{Introduction}

Natural rubber latex is obtained from the botanical source Hevea brasiliensis and is the name commonly used to denote a stable colloidal dispersion of a polymeric substance of cis-1,4 polyisoprene of high molecular mass in an aqueous medium [1]. Since rubber latex is an exudate of the plant's cytosol, it contains many of the substances found naturally in the tree: carbohydrates, proteins, fats, and inorganic and organic salts.

The freshly tapped natural rubber latex, also called

*Corresponding author. Fax: +34-91-351-0475.

E-mail address: cbarbas@ceu.es (C. Barbas). field latex, contains a rubber content of about $33 \%$. As soon as possible, the latex is temporarily preserved with ammonia (the quantity depending on the delay between processing into concentrate and harvesting the crop) in order to prevent bacterial contamination or at least to limit its effects.

The raw field latex is either centrifuged or creamed to concentrate the latex to about $60 \%$ dry rubber content for use. Centrifugation is the most common method for the production of latex concentrate [2]. There are only two types made on a large scale: HA (high-ammonia) latex, preserved with $0.7 \%$ ammonia and LA-TZ (low-ammonia) latex, preserved with $0.2 \%$ ammonia $+0.025 \%$ tetramethylthiuram disulphide (TMTD) $\mathrm{ZnO}+0.04-$ 
$0.05 \%$ lauric acid as ammonium laurate [3]. Double centrifuged (DC) latex is a highly purified latex concentrate prepared by re-centrifuging the first centrifuged latex, which has been suitably diluted. As a result, the non-rubber constituents are further reduced.

Natural rubber continues to be of great economic importance for industry. Nowadays, the increasing use of latex products in the healthcare area has also increased new scientific controls of raw materials and manufactured products in order to maintain higher standards in quality control. On the other hand, it has become more necessary to develop other technical approaches to improve the quality of the rubber-culture in order to maintain and develop its competitivity against synthetic rubber.

Since field latex is a rich environment for microbial growth, in the absence of adequate preservation, the carbohydrates become microbiologically oxidised to the so-called volatile fatty acids (VFAs), which comprise mainly formic, acetic and propionic acids [1]. Fig. 1 shows a scheme of the fermentation process. For this reason their measurement gives an indication of the extent of microorganic activity which has occurred since the latex exuded from the tree. These matters are of considerable industrial importance, because several months may elapse between the production of preserved and concen-

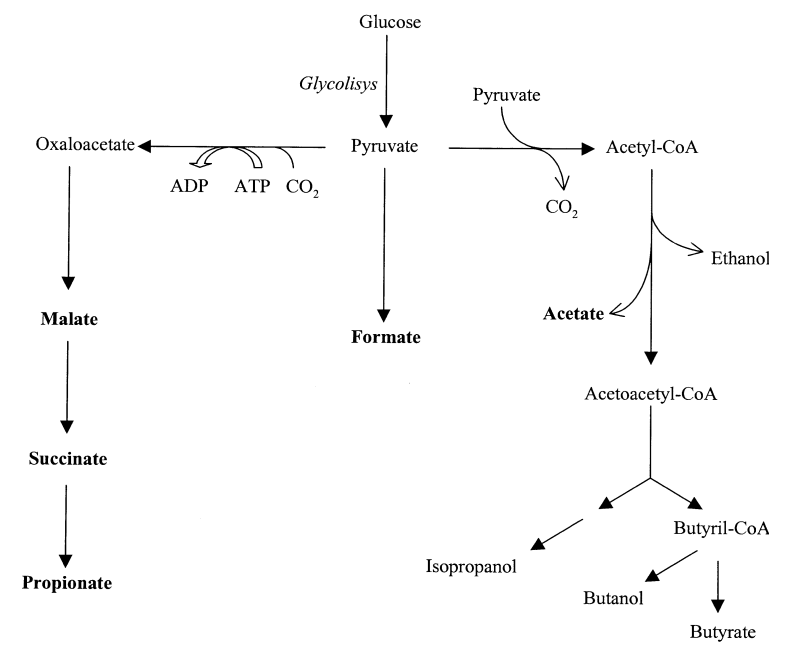

Fig. 1. Fermentation pathways due to bacterial action upon glucose. trated natural rubber latex and its eventual use. Volatile fatty acid (VFA) number, measured in the steam of a quantity of latex, is a classical test in quality control. The VFA number is defined as the number of grams of potassium hydroxide equivalent to the anions present as salts of steam-volatile acids in a quantity of latex which contains $100 \mathrm{~g}$ of total solids. The ionic content of latex is of continuing interest since it is believed to contribute to the properties and especially the processing of the latex [4], at least for natural rubber latices of low VFAs, it is thought that the salts of non-volatile fatty acids (NVAs) significantly influence latex mechanical stability [2]. According to different sources [4-8], the principal non-volatile organic acids found in natural rubber latex are citric and malic. Minor amounts of trans-aconitic, fumaric, glycolic, succinic, quinic and glutaric may also be present. Their concentration also gives us information about the quality of latex, because they have significant influence in the mechanical stability [1].

Furthermore, some of these acids mediate the biogenesis of polyisoprenes and their measurement can help in the study of the metabolism of the plants which produce them, since their biological function is largely unknown.

Previously reported works on the measurement of short-chain organic acids in natural rubber latex were carried out using ion chromatography $[4,6,9]$.

Capillary electrophoresis (CE) has proved to be a good choice for investigation of samples in aqueous media, since usually no more than a simple dilution of samples is needed. Our research group has developed several methods for the separation of organic acids into diverse matrices [10-13] with direct UV detection at $200 \mathrm{~nm}$ where the absorbance has a maximum. This low wavelength can be employed when working with non UV-absorbing aqueous buffers. A CE method was developed and validated by our group to identify the short-chain organic acids present in sera of natural rubber latex directly without any sample pre-treatment more than coagulation [14].

The aim of the present work was to apply that method for measuring a number of natural rubber latex samples of different characteristics and evaluate these acids trying to find patterns to establish ranges useful for quality control. 


\section{Materials and methods}

\subsection{Instrumentation}

The separation was performed on a P/ACE 5500 (Beckman) CE instrument with UV detection at 200 $\mathrm{nm}$. The injection was by pressure $(0.035 \mathrm{bar})$ for 5 $\mathrm{s}$. The separation was carried out with an uncoated fused-silica capillary $(57 \mathrm{~cm} \times 50 \mu \mathrm{m}$ I.D.) and was operated at $-10 \mathrm{kV}$ potential. Temperature was maintained at $25^{\circ} \mathrm{C}$. The separation buffer was prepared with $0.5 \mathrm{M} \mathrm{H}_{3} \mathrm{PO}_{4}, 0.5 \mathrm{~m} M \mathrm{CTAB}$, as cationic surfactant to avoid electroosmotic flow, and $\mathrm{pH}$ adjusted by adding $\mathrm{NaOH}$ at 6.25 .

\subsection{Chemicals}

Standards were obtained from Sigma (St. Louis, MO, USA). Phosphoric acid 85\% was from Merck (Darmstadt, Germany), sodium hydroxide from Panreac (Madrid, Spain) and organic solvents from Scharlau (Barcelona, Spain).

\subsection{Samples}

Samples of different origins were assayed in order to compare the validity of the results. Samples came from Thailand, Africa and Guatemala. One of them was field latex (not preserved) and the remaining samples were centrifuged once and double centrifuged. They were directly imported and were representative of commercial material.

\subsection{Coagulation}

For coagulation, two conditions were employed, which previously demonstrated to be useful when analysing the samples by CE [14]. One of them it was chemical destabilisation with the addition of chemical agents such as phosphoric acid and the other was physical destabilisation by freezing. In the acidic coagulation, $10 \% \quad \mathrm{H}_{3} \mathrm{PO}_{4}(\mathrm{v} / \mathrm{v})$ was mixed with the latex sample $1: 2(\mathrm{v} / \mathrm{w} ; \mathrm{ml} / \mathrm{g})$, stirred vigorously and the serum was filtered through 0.45 $\mu \mathrm{m}$ nylon filters and diluted 1:2 (v/v) with purified water prior to analysis. In the second method, samples were simply kept at $-20^{\circ}$ overnight. The next day, once the samples had reached room temperature the serum was filtered and measured without dilution.

\subsection{VFA contents}

The VFA number was determined by the ISO 506 method, i.e. by coagulation and acidification followed by steam distillation [15].

Then, $50 \mathrm{~g}$ of latex test sample, to which $50 \mathrm{ml}$ of an $30 \%(\mathrm{w} / \mathrm{v})$ ammonium sulphate solution were added, was heated in a beaker either on a steam bath or in water bath at $70^{\circ} \mathrm{C}$. It was stirred until coagulation occurred and it was then covered and heated for a further $15 \mathrm{~min}$. The exuded aqueous phase was decanted through a dry filter. As much additional aqueous phase as possible was then extracted by pressing with a pestle and mortar. This was filtered through the same filter. After acidification with $5 \mathrm{ml}$ of an aqueous $30 \%(\mathrm{v} / \mathrm{v})$ sulphuric acid solution, a $10 \mathrm{ml}$ aliquot was steam-distilled until $100 \mathrm{ml}$ of distillate had been collected. The distillate was tritrated with a $0.01-0.005 M$ barium hydroxide solution using phenolphthalein.

\section{Results and discussion}

With acidic coagulation of samples a system peak appears. It is due to the high electric field created by the elevated phosphoric acid concentration in samples, which is needed to coagulate the latex. This method is more rapid than the physical destabilisation and it would be the method of choice when testing latex for rapid quality control. The second method of coagulation is longer but peaks are higher and sharp, because the lower ionic strength favours the stacking effect and no dilution is needed. If time were not a parameter to be considered it would be the best choice. The electropherograms for two samples of the same latex in both conditions are shown in Fig. 2. Peaks were identified by comparison with the retention times of standards and by spiking. As can be observed, the profile of serum obtained by freezing gave higher peaks. It provides better sensitivity, which is necessary when studying the whole group of acids in detail. A new peak appeared overlapping with nitrate, which was iden- 


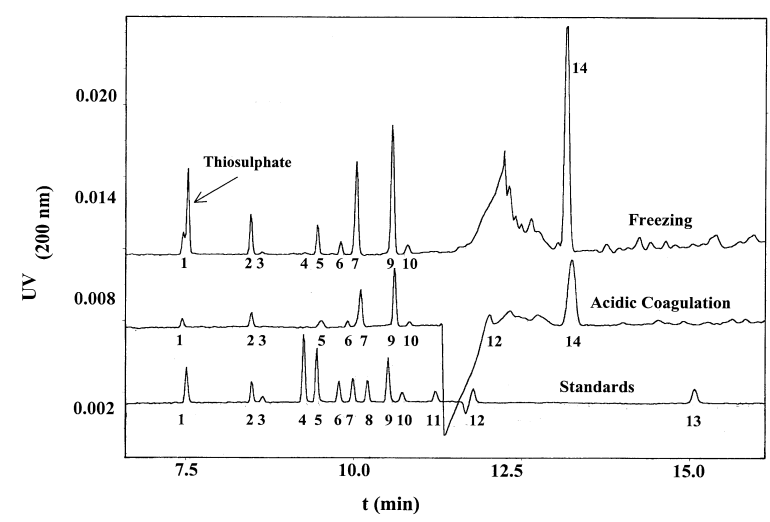

Fig. 2. Electropherogram of serum of latex in both destabilisation processes. Peaks in order correspond to: (1) nitrate; (2) oxalate; (3) formate; (4) fumarate; (5) aconitate; (6) succinate; (7) malate; (8) glutarate; (9) citrate; (10) acetate; (11) glycolate; (12) propionate; (13) quinate; and (14) unknown. Buffer, $0.5 M \mathrm{H}_{3} \mathrm{PO}_{4}$ with $\mathrm{pH}$ adjusted by adding $\mathrm{NaOH}$ at 6.25 and $0.5 \mathrm{~m} M$ CTAB. UV detection at $200 \mathrm{~nm}$. Injection by pressure $(0.035$ bar) for $5 \mathrm{~s}$. Uncoated fused-silica capillary $(57 \mathrm{~cm} \times 50 \mu \mathrm{m}$ I.D.), $-10 \mathrm{kV}$ applied voltage. Temperature $25^{\circ} \mathrm{C}$.

tified as thiosulphate, but this peak, was lacking in acidic media, because it is not stable in acidic media.

Table 1 shows the results obtained for short-chain organic acid contents of 16 samples coming from Thailand (T), Guatemala (G) and Africa (A) and their VFA numbers. These samples underwent different treatments. Only one of them (sample A), is field latex without any treatment (NT), five of them (samples B-F) were centrifuged once and 10 sam- ples were double centrifuged ( $\mathrm{G}-\mathrm{P})$. Quantification was performed by processing standards of the organic acids in the same assay.

Field latex (A), without any preserving agent added to avoid fermentation, presented high VFA values which was related with high levels of formic and acetic acids measured by CE, but also higher values of succinic acid and lower value of malic acid than the same sample centrifuged once and preserved (C).

Samples B and G came from Thailand but they underwent different treatments. Sample B was centrifuged once and sample $\mathrm{G}$ double centrifuged. As was expected the organic acid content in sample $G$ was lower than in sample $B$, because $G$ was double centrifuged. In general terms, latex centrifuged once presented higher VFAs and higher values for most of the individual acids than latex double centrifuged, because organic acids are partially eliminated with the serum during the process. This effect is not related to the quality of the preservation process.

Samples D and E, shown in Fig. 3, came from the same origin and treatment, but one of them, sample $\mathrm{E}$, had undergone a poor conservation process. This sample showed an increase in most of the organic acids, mainly succinic and acetic acids but also a decrease in malic acid content.

It has been observed that when VFAs rise, succinic acid increases and malic acid decreases (samples A, $\mathrm{B}, \mathrm{E}, \mathrm{F}, \mathrm{L}, \mathrm{P})$. This is usually associated with poor conservation of latex and poor mechanical stability.

Table 1

Short-chain organic acid content in sera of different types of latices

\begin{tabular}{|c|c|c|c|c|c|c|c|c|c|c|c|c|c|c|c|c|}
\hline Samples & A & B & $\mathrm{C}$ & $\mathrm{D}$ & E & $\mathrm{F}$ & G & $\mathrm{H}$ & I & $\mathrm{J}$ & K & $\mathrm{L}$ & M & $\mathrm{N}$ & 0 & $\mathrm{P}$ \\
\hline Origin & G & $\mathrm{T}$ & G & G & G & A & $\mathrm{T}$ & G & G & G & G & G & G & G & G & G \\
\hline Concentration process & NT & $\mathrm{CO}$ & $\mathrm{CO}$ & $\mathrm{CO}$ & $\mathrm{CO}$ & $\mathrm{CO}$ & DC & DC & $\mathrm{DC}$ & $\mathrm{DC}$ & DC & DC & DC & $\mathrm{DC}$ & DC & DC \\
\hline \multirow[t]{2}{*}{ VFA No. } & 0.08 & 0.043 & 0.0125 & 0.011 & 0.048 & 0.095 & 0.018 & 0.02 & 0.018 & 0.021 & 0.019 & 0.041 & 0.026 & 0.021 & 0.014 & 0.082 \\
\hline & \multicolumn{16}{|c|}{ mmoles $1^{-1}$ serum } \\
\hline Oxalate & 0.53 & 1.83 & 0.67 & 2.41 & 3.97 & 0.91 & 0.82 & 0.92 & 1.02 & 1.06 & 0.41 & 0.60 & 0.54 & 0.48 & 0.34 & 0.40 \\
\hline Formate & 9.50 & 3.31 & n.d & 1.18 & 3.97 & 1.70 & 3.13 & 2.25 & 2.64 & 2.37 & 1.26 & 2.62 & 1.91 & 1.81 & n.d & n.d \\
\hline Fumarate & n.d & n.d & 0.29 & 0.01 & 0.04 & n.d & n.d & 0.02 & 0.02 & 0.01 & 0.01 & 0.01 & 0.02 & 0.01 & n.d & n.d \\
\hline Aconitate & 0.23 & 0.29 & 0.29 & 0.37 & 0.57 & 0.23 & 0.14 & 0.14 & 0.13 & 0.13 & 0.06 & 0.11 & 0.07 & 0.06 & 0.07 & 0.07 \\
\hline Succinate & 23.10 & 5.06 & 0.76 & 5.30 & 39.46 & 9.63 & 1.51 & 2.04 & 3.82 & 4.00 & 1.15 & 3.49 & 1.92 & 1.66 & 1.02 & 2.04 \\
\hline Malate & 1.80 & 8.36 & 14.68 & 27.86 & 6.49 & 0.67 & 6.27 & 7.36 & 6.63 & 7.09 & 3.03 & 2.11 & 3.74 & 3.40 & 1.84 & 1.64 \\
\hline Citrate & 8.08 & 12.08 & 10.51 & 15.57 & 17.99 & 4.13 & 7.36 & 4.46 & 5.81 & 6.35 & 2.350 & 2.53 & 2.72 & 2.39 & 1.84 & 1.93 \\
\hline Acetate & 24.50 & 8.38 & 2.66 & 7.46 & 29.54 & 9.76 & 2.95 & 2.58 & 3.95 & 4.09 & 1.51 & 4.43 & 2.82 & 2.73 & 1.35 & 2.57 \\
\hline Succinate/malate & 12.84 & 0.61 & 0.05 & 0.19 & 6.08 & 14.42 & 0.24 & 0.28 & 0.58 & 0.56 & 0.38 & 1.66 & 0.51 & 0.49 & 0.55 & 1.25 \\
\hline
\end{tabular}




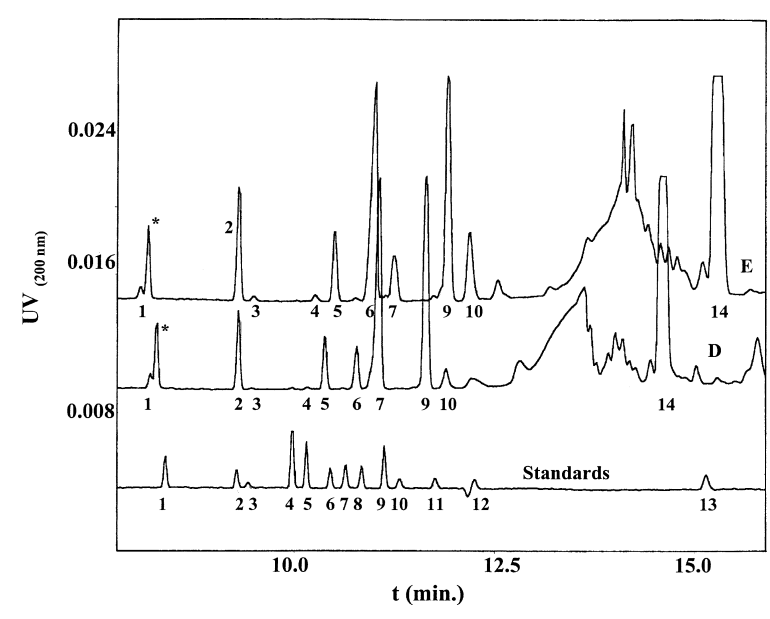

Fig. 3. Electropherogram of sera of Guatemala latex. Peaks correspond to: (1) nitrate; (*) thiosulphate; (2) oxalate; (3) formate; (4) fumarate; (5) aconitate; (6) succinate; (7) malate; (8) glutarate; (9) citrate; (10) acetate; (11) glycolate; (12) propionate; (13) quinate; and (14) unknown. Buffer, $0.5 \mathrm{M} \mathrm{H}_{3} \mathrm{PO}_{4}$ with $\mathrm{pH}$ adjusted by adding $\mathrm{NaOH}$ at 6.25 and $0.5 \mathrm{~m} M$ CTAB. UV detection at $200 \mathrm{~nm}$. Injection by pressure $(0.035$ bar) for $5 \mathrm{~s}$. Uncoated fused-silica capillary $(57 \mathrm{~cm} \times 50 \mu \mathrm{m}$ I.D. $),-10 \mathrm{kV}$ potential. Temperature $25^{\circ} \mathrm{C}$.

It is important to remember the fermentation pathways due to bacterial action upon glucose (Fig. 1). In that process VFAs (formic, acetic and propionic acids) are formed. But, moreover, malic acid is transformed into succinic acid and therefore, the increased ratio could be justified. These data are in agreement with those found by Pendle [6] over LA-TZ stored for 87 days and measured by ion chromatography. Measurements showed an increase in citric, formic and succinic acids and a decrease in malic acid. After analysing data from different sources the author concludes that well-preserved latices should not show significant increases in VFA numbers under normal storage conditions. Where abnormal rises do occur, they are most likely to be caused by poor storage conditions. Storage in unclean tanks or drums, improper closure of vessels permitting loss of ammonia, or exposure to excessively high temperatures $\left(>40^{\circ} \mathrm{C}\right)$ can all cause an increase in VFA numbers and a consequent loss of stability.

In the assayed samples, it has been shown that poorly-preserved latex with VFA numbers over 0.03 presented higher succinic acid and lower malic acid
Table 2

Short-chain organic acid content in sera of different types of mixtures for latex manufacturing

\begin{tabular}{llc}
\hline Samples & $\mathrm{Q}$ & $\mathrm{R}$ \\
\hline Origin & $\mathrm{G}$ & $\mathrm{G}$ \\
Concentration process & $\mathrm{DC}$ & $\mathrm{DC}$ \\
& \multicolumn{2}{c}{$\mathrm{mmoles}^{-1}$ serum } \\
\cline { 2 - 3 } Oxalate & 0.24 & 0.27 \\
Formate & 1.42 & 1.11 \\
Fumarate & $\mathrm{nd}$ & $\mathrm{nd}$ \\
Aconitate & 0.02 & 0.05 \\
Succinate & 1.36 & 0.51 \\
Malate & 1.03 & 2.39 \\
Citrate & 0.95 & 1.37 \\
Acetate & 2.62 & 1.05 \\
Succinate/malate & 0.61 & 12.84 \\
\hline
\end{tabular}

contents. The succinic to malic ratio may be an important parameter because it cannot be altered by dilution or similar processes such as the traditional VFA index, used to determine the quality of latex. Ratios for succinic to malic lower than 0.6 have been found for well-preserved latex and higher than 0.6 for poorly-preserved latex. Therefore, it has been proved that, in absence of adequate conservation, the organic acid content in sera of natural latex increases and the concentration processes have an effect on these acids.

The method was also applied to two mixtures $(\mathrm{Q}$ and $\mathrm{R}$ ) composed of natural rubber latex and the usual compounds added during manufacturing used for dipped rubber products, the results obtained for short-chain organic acids content are shown in Table 2. One of them, mixture $\mathrm{R}$, had shown abnormal behaviour in manufacturing (bubbles and bad unfold). In Fig. 4, it is compared with a similar mixture of standard behaviour (sample Q). Again succinic and acetic acid levels increased, and malic acid decreased in mixture $\mathrm{R}$.

\section{Conclusion}

There is a relationship found between an increase in the succinic acid to malic acid ratio and the traditional VFA index on the one side, and a lw quality of latex on the other. It has been proven that, in the absence of adequate preservation, the organic 


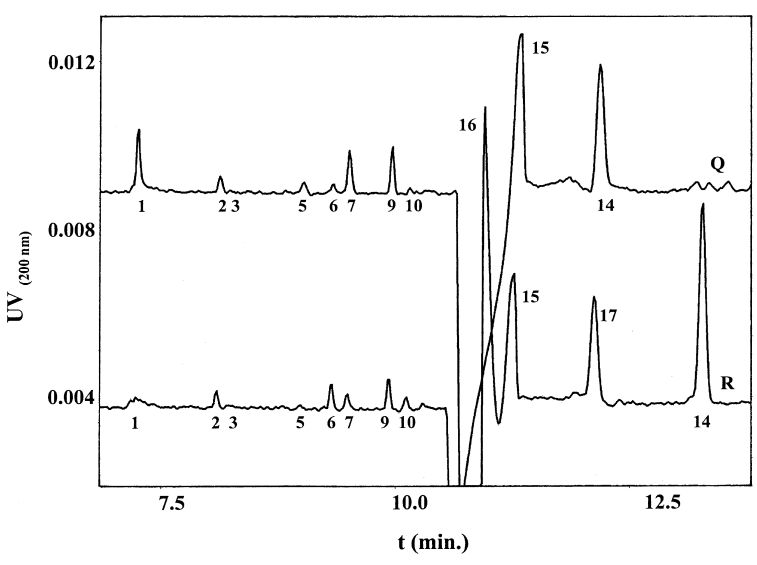

Fig. 4. Electropherogram of sera of mixtures. Peaks correspond to: (1) nitrate; (2) oxalate; (3) formate; (5) aconitate; (6) succinate; (7) malate; (9) citrate; (10) acetate; (14) unknown; (15) unknown; and (16) unknown. Buffer, $0.5 \mathrm{M} \mathrm{H}_{3} \mathrm{PO}_{4}$ with $\mathrm{pH}$ adjusted by adding $\mathrm{NaOH}$ at 6.25 and $0.5 \mathrm{~m} M$ CTAB. UV detection at $200 \mathrm{~nm}$. Injection by pressure $(0.035$ bar) for $5 \mathrm{~s}$. Uncoated fused-silica capillary $(57 \mathrm{~cm} \times 50 \mu \mathrm{m}$ I.D.), $-10 \mathrm{kV}$ applied voltage. Temperature $25^{\circ} \mathrm{C}$.

acid content in sera of natural latex increases and the concentration processes have an effect on these acids. CE proved to be an excellent tool in the quality control of natural rubber latex.

\section{References}

[1] D.C. Blackley, 2nd ed, Polymer Latices - Science and Technology, Fundamental Principles, Vol. 1, Chapman and Hall, London, 1997.

[2] D.C. Blackley, 2nd ed, Polymer Latices - Science and Technology, Types of Latices, Vol. 2, Chapman and Hall, London, 1997.

[3] Web page, available at http://www.rubber-stichting.ind. tno.nl.

[4] R.C. Crafts, Polym. Testing 5 (1985) 193.

[5] K.O. Calvert, Plastics Rubber: Mater. Appl. 2 (1977) 59.

[6] T.D. Pendle, Rubber Chem. Technol. 63 (1990) 234.

[7] R.T. Davies, T.D. Pendle, Rubber Dev. 44 (1991) 94.

[8] R.C. Crafts, A.D.T. Gorton, T.D. Pendle, Rubber Dev. 16 (Part 1) (1985) 12.

[9] R.C. Crafts, J.E. Davey, G.P. McSweeney, I.S. Stephens, J. Nat. Rubber Res. 5 (1990) 275.

[10] C. Barbas, N. Adeva, R. Aguilar, M. Rosillo, T. Rubio, M. Castro, Clin. Chem. 44 (1998) 1340.

[11] A. García, C. Barbas, R. Aguilar, M. Castro, Clin. Chem. 44 (1998) 1905.

[12] C. Barbas, J.A. Lucas, F.J. Gutiérrez-Mañero, Phytochem. Anal. 10 (1999) 55.

[13] L. Saavedra, A. García, C. Barbas, J. Chromatogr. A 881 (2000) 395.

[14] V. Galli, N. Olmo, C. Barbas, J. Chromatogr. A 894 (2000) 135.

[15] ISO Standard 506: Rubber Latex, Natural, Concentrate Determination of Volatile Fatty Acid Number, 1992. 